\title{
Capsule Commentary on Chodos et al., Hospitalization-Associated Disability in Adults Admitted to a Safety Net Hospital
}

\author{
Kirk V. Shepard II, MD \\ Department of Internal Medicine, Medical College of Wisconsin, Milwaukee, WI, USA.
}

$\mathrm{J}$ Gen Intern Med 30(12): 1846

DOI: $10.1007 / \mathrm{s} 11606-015-3477-1$

(c) Society of General Internal Medicine 2015

$\mathrm{T}$ he study by Chodos et al. ${ }^{1}$ investigated hospitalizationassociated disability, which is new or additional disability in activities of daily living at hospital discharge compared to pre-admission baseline, ${ }^{2}$ and its association with age in adults aged 55 and older hospitalized at a safety net hospital. Adults treated at safety net hospitals also tend to be more prone to hospitalization-associated disability due to a concentration of risk factors, including minority race, functional impairment, low socioeconomic status, substance abuse, and homelessness. ${ }^{3,4}$ The authors found that hospitalization-associated disability was common, occurring in one-fourth of adults aged 55-59, only very slightly higher among those aged 70-79 $(30.3 \%)$, and highest in adults aged 80 and above $(61.7 \%)$. They concluded that interventions to reduce hospitalizationassociated disability should be considered in hospitalized adults as young as 55 , but particularly among those 70 years and older at safety net hospitals.

The reported associations may have been confounded by several factors. First, the study relied on patient self-reporting of functional status before, at admission, and after hospitalization. While seemingly unavoidable in assessing functional status before hospitalization, admission and 30-day follow-up functional status could have been more accurately evaluated with expert clinician assessment. Additionally, the selfreporting of functional status 2 weeks prior to admission was subject to recall bias. Moreover, the authors do not differentiate between disability developed from the inciting illness that led to hospitalization versus disability from the hospitalization itself. Lastly, while the authors described hospitalizationassociated disability related to patient age and number of comorbidities separately, they do comment on the whether a particular age group had more comorbidities than another, which may influence the likelihood of disability within a particular age group.

Disability in functional status threatens quality of life and predicts higher resource utilization and mortality. ${ }^{5}$ Chodos et al. provide evidence for expanding age criteria for interventions to target and reduce hospitalization-associated disability in hospitalized adults at safety net hospitals to as young as 55 years. What remains uncertain and needs further research is exactly which interventions may prove useful in preventing hospital-related disability.

Conflict of Interest: The author declares that he does not have a conflict of interest.

Corresponding Author: Kirk V. Shepard, II, MD; Department of Internal MedicineMedical College of Wisconsin, Milwaukee, WI, USA (e-mail: kishepard@mcw.edu).

\section{REFERENCES}

1. Chodos AH, Kushel MB, Greyson SR, et al. Hospitalization-Associated Disability in Adults Admitted to a Safety Net Hospital. J Gen Intern Med. 2015. doi:10.1007/s11606-015-3395-2.

2. Gill TM, Allore HG, Gahbauer EA, Murphy TE. Change in disability after hospitalization or restricted activity in older persons. JAMA. 2010;304(17): 1919-28.

3. Brown RT, et al. Geriatric syndromes in older homeless adults. J Gen Intern Med. 2012;27(1):16-22.

4. Minkler M, Fuller-Thomson E, Guralink JM. Gradient of disability across the socioeconomic spectrum in the United States. N Engl J Med. 2006;355(7):695-703.

5. Covinsky KE, Justice AC, Rosenthal GE, et al. Measuring prognosis and case mix in hospitalized elders. The importance of functional status. J Gen Intern Med. 1997;12(4):203-8. 